\title{
S@J-Wiki: A Collaborative Semantic Wiki to Manage Juridical Knowledge
}

\author{
Amaigarou Noureddin \\ Laboratory of Computer Science Operational \\ Research and Applied Statistics, \\ Abdelmalek Essaadi University, Faculty of Science, \\ Tetouan, Morocco B.P. 209 Martil, Morocco
}

\author{
Mohamed Khaldi \\ Laboratory of Computer Science Operational \\ Research and Applied Statistics, \\ Abdelmalek Essaadi University, Higher Normal \\ School, Tetouan, Morocco B.P. 209 Martil, Morocco
}

\begin{abstract}
This article describes the architecture and the principles of a semantic wiki for collaborative knowledge management in an environment of sharing and juridical learning, our wiki is a multi-agent platform based on ontologies, has the goal of identification, extraction, validation and ends with the reuse of knowledge circulating in the juridical field. Outstanding features of our wiki is its support for collaborative knowledge engineering, its ease of use, its support for different levels of formalization from informal texts to formal ontologies, and its sophisticated, interactive user interface.
\end{abstract}

\section{General Terms}

E-learning, Intelligent system, engineering ontologies

\section{Keywords}

Semantic Wiki, Semantic Web, knowledge, multi-agent systems (MAS), ontologies.

\section{INTRODUCTION}

The juridical business is based on knowledge, which requires not only access to information but also the ability to use it to innovate and act in the promulgation of juridical resolutions. Thus, management of juridical knowledge contributes to the modernization of justice as it established more efficient working methods in the exercise of the legal function.

Knowledge management can identify and capitalize on the knowledge ness of the enterprise to organize and disseminate. This paper proposes a system of knowledge management based on ontologies and on a multi-agent system to resolve the problem of capitalization and reuse of knowledge from many different sources that Knowledge management system aims to help actors and learners in an environment of sharing, developing intellectual memory of the juridical body.

In our case it is to provide a semantic wiki for sharing and management of legal knowledge (S @ J-Wiki). Our wiki is a multi-agent system based on a life cycle of four stages This life cycle has phases: identification, extraction, validation and ends with knowledge reuse. Each phase is the goal of an organization of agents.

We can present the process of knowledge management in our wiki in this model. [1]. The model contains 4 process:

The localization process: the problems surrounding the identification of explicit and tacit knowledge needed to carry out the activities of the company.

The preservation process: when knowledge is explainable, it is necessary to acquire to holders of knowledge, model, formalize and maintain.

The valuation process: aims to make knowledge accessible according to certain rules of confidentiality and security, distribute, share, exploit / reuse, combine and create new knowledge.

The updating process: assessing knowledge, updates and richer.

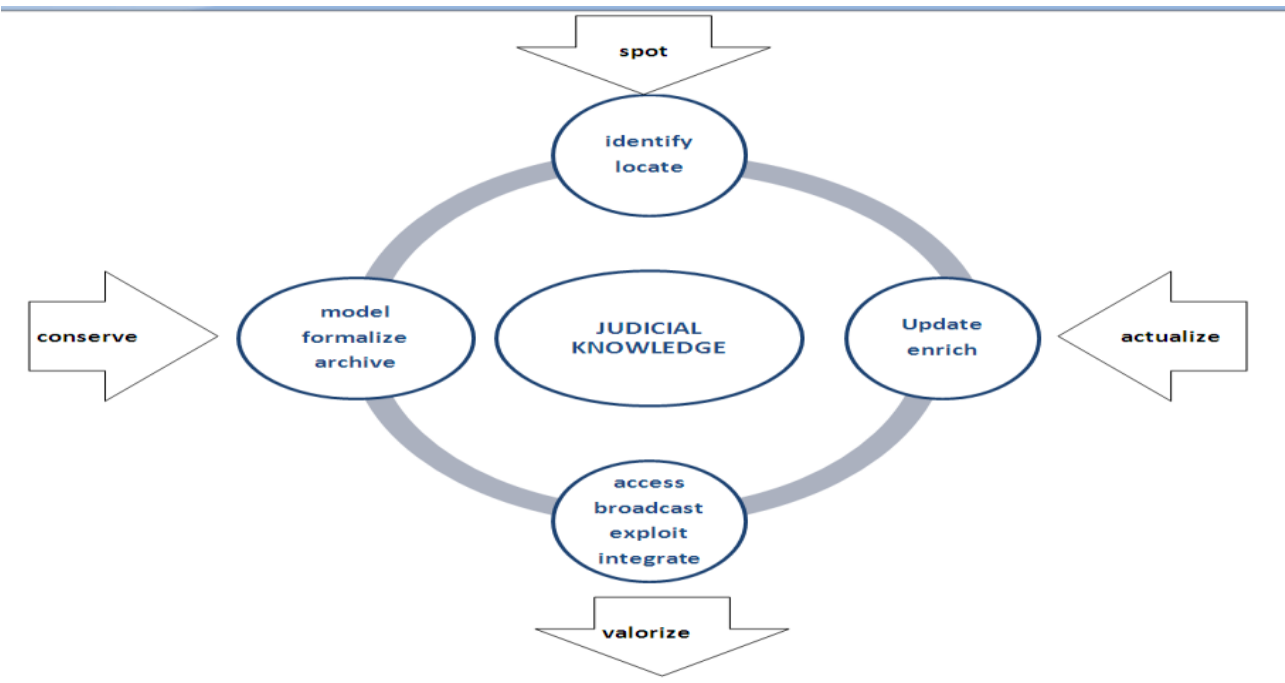

Fig 1: Lifecycle process of knowledge management 


\section{WHY A SEMANTIC WIKI?}

\subsection{Semantic web}

"The semantic Web is an extension of the current web in which information is given well defined meaning, better enabling computers and people to work in cooperation" [2].

Like the classic web, the Semantic Web consists of resources and links. However, now the resources and links can have types that define the concepts that give a little more information about their contents. For example, some links may say that a resource is a version of another resource or written by a resource that describes a person or resource contains software that depends on other software. Here we wrote the guy inside the node, and stressed the similarities. Types are generally defined by a link type node with a URI, as shown in the detailed model in Figure 2.

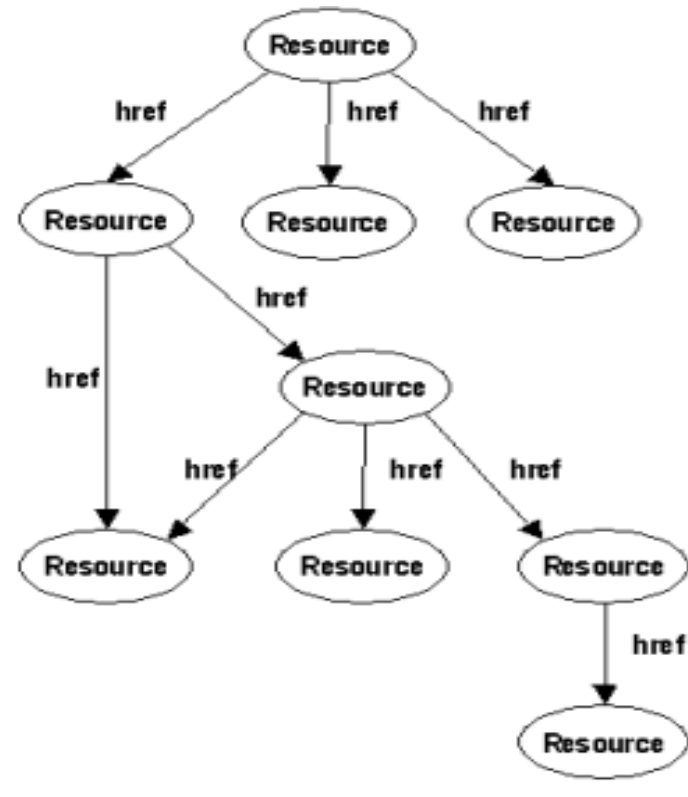

a) Current Web

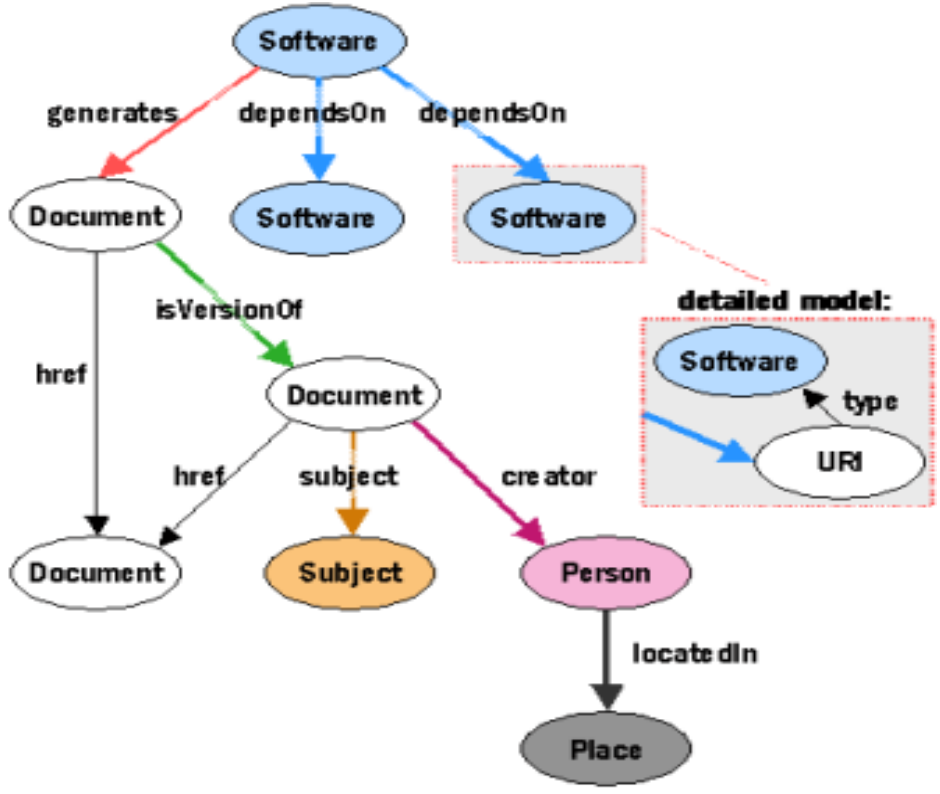

b) Semantic Web

Fig 2: W3C Semantic Web Activity [3]

\subsection{Semantic Wiki}

A wiki is a website that allows remote collaborative authoring of information and publishing hypertext content. Leuf \& Cunningham [4] are the first who suggested a website where users can create, edit, convert and share links from their browser in a very simple manner. Wikis have become popular collaboration tools on the Web, and many active online communities use wikis to publish or exchange information.

For most wikis, public or private, the main goals are to organize information, to collect and share. Wikis are generally considered tools to manage content online in a quick and easy way. Schaffert [5] lists the characteristics of a wiki system as follows:

- It allows editing via browser;

- It has a hypertext format in more than the simplified syntax of the wiki to make it usable by all Internet users;

- Manages a recovery mechanism ie that it is able to update the changes in the content whenever they are stored;

- $\quad$ Access is free, anyone can write to the wiki;

- Manages collaborative editing whether someone create an article, everyone can extend this article;
- It offers a strong bond, all wiki pages are linked with others using hyperlinks to each;

- It has a feature of reliable and intelligent search the contents of all pages stored;

The semantic wiki bears much potential in many application areas such as Schaffert [5] lists a number of scenarios for semantic wiki:

- ontological engineering, where domain experts and knowledge engineers working together can be supported by a semantic wiki. The experts will have an easy way to get the knowledge they can all work together to work on the ontology and knowledge can be scalable and formalized.

- Systems knowledge management must combine content creation and easy creation of knowledge in order to retrieve related information. Traditional Wikis can be used for the former, while semantic wiki adds support for it.

- Educational environments can be supported by semantic wiki where content can be created, modified and linked to the relevant learning materials. In addition, structured and interconnected content in a semantic wiki can support self-directed learning. 
In our work, we are more in the case of ontology engineering. Thus, we use a semantic approach and exploit Innovation Wiki to provide a system that can facilitate creativity and formalize ideas in learning activities by facilitating knowledge sharing, updating and evaluation.

\section{S@J-WIKI: A SEMANTIC WIKI JURIDICAL}

S @ J-wiki is a wiki system prototype under development as part of our research. S @ J-wiki serves several purposes:

1- can be used to annotate existing data with terms to improve search and navigation;

2- it can be used to create instance data based on an existing ontology;

3- can be used as a tool for creating and editing ontologies.

All three goals can be monitored simultaneously, perhaps by users with different roles and different levels of experience in knowledge engineering. Indeed, many engineering knowledge more complex tasks probably require this kind of collaboration.

In addition, S @ J-Wiki refers to the following objectives::

- The syntax compatibility and appearance with existing systems (currently Wikipedia); allowing users to take existing knowledge (eg Wikipedia), import in S@J-Wiki, and begin the formalization of knowledge right away.

- Compatibility with existing Semantic Web technologies; Currently, S@J-Wiki uses RDF and OWL to store and reason with formal knowledge.

- Immediate exploitation of formal knowledge for navigation and existing publishing; Users should get an instant reward for the extra effort they put into formalizing their knowledge.

- Easy access to common tasks; but still give users the functionality and complexity if they wish.
- feeling of an application, not a website; User interface should support the user beyond "wiki syntax" by providing a modern graphical interaction with the system (eg, WYSIWYG editing).

Knowledge creation in S @ J-Wiki is supposed to be an open community process where experts from different fields can work together.

An expert in the field could begin by describing domain knowledge in S @ J-Wiki, to the point where its expertise in knowledge technologies is not enough to do more. If necessary, an expert in knowledge engineering could then participate and help create more formal representations.

Knowledge in our wiki is presented by ontologies, and his creation, archiving, representation and evaluation done by collaborated agent in a multi-agent system.

\section{ONTOLOGIES AND MULTI-AGENT SYSTEM}

Systems management of heterogeneous and distributed knowledge are quite complex to manage, and require significant ongoing user intervention. To overcome these drawbacks, one of the assumptions of our work is to use the paradigm of multi-agent systems. Indeed, the agents paradigm has proved useful to manage this complexity. A knowledge management system (KMS) is used in an organization to manage the explicit [14] or tacit knowledge. This means that knowledge is clearly identified in a written document (eg, the length of the seat tube $=85 \mathrm{~cm}$ ) or from a software (CAD tool, calculation, etc.).

\subsection{Approaches agents for knowledge management}

There are many platforms that describe the cooperation of agent to solve complex problems related to knowledge as FRODO [14] , CoMMA [15], Edamok [16], Kraft [17] Musette [18], Katras [19], MAEKMS and [20]. We then present a descriptive table some systems mentioned above:

Table 1 : Comparison of SMA for knowledge management

\begin{tabular}{|c|c|c|c|c|c|c|c|}
\hline Approach & Kraft & Frodo & Comma & KATRAS & FU Rui-xue & Yang & MAEKMS \\
\hline \multicolumn{8}{|l|}{ Step } \\
\hline \multicolumn{8}{|l|}{ Identification } \\
\hline Acquisition & $\mathrm{X}$ & $\mathrm{X}$ & $\mathrm{X}$ & $X$ & $X$ & $\mathrm{X}$ & $X$ \\
\hline Formalization & & & $\mathrm{X}$ & $X$ & & & $X$ \\
\hline Organization & $\mathrm{X}$ & & & $\mathrm{X}$ & & & $\mathrm{X}$ \\
\hline \multicolumn{8}{|l|}{ Research } \\
\hline Extraction & $\mathrm{X}$ & $X$ & $X$ & & $\mathrm{X}$ & $X$ & $X$ \\
\hline Translation / Tran & $\mathrm{X}$ & & & & $\mathrm{X}$ & & \\
\hline \multicolumn{8}{|l|}{ Sformation } \\
\hline Annotation & & & $\mathrm{X}$ & $\mathrm{X}$ & $\mathrm{X}$ & & \\
\hline Storage & $\mathrm{X}$ & $\mathrm{X}$ & $\mathrm{X}$ & $\mathrm{X}$ & $\mathrm{X}$ & $\mathrm{X}$ & $X$ \\
\hline Presentation & $\mathrm{X}$ & $\mathrm{X}$ & $\mathrm{X}$ & & $\mathrm{X}$ & $\mathrm{X}$ & $X$ \\
\hline \multicolumn{8}{|l|}{ Distribution } \\
\hline Evaluation & & & & $\mathrm{X}$ & $\mathrm{X}$ & & \\
\hline Evolution & & $\mathrm{X}$ & & $\mathrm{X}$ & $\mathrm{X}$ & & $X$ \\
\hline Distribution & $X$ & $\mathrm{X}$ & & $\mathrm{X}$ & $\mathrm{X}$ & & $X$ \\
\hline Reuse & $\mathrm{X}$ & $\mathrm{X}$ & & $\mathrm{X}$ & & & $\mathrm{X}$ \\
\hline Storage Base & $\begin{array}{l}\text { Knowledge } \\
\text { Base }\end{array}$ & $\begin{array}{l}\text { Organizational } \\
\text { Memories }\end{array}$ & $\begin{array}{c}\text { Memory } \\
\text { Project }\end{array}$ & $\begin{array}{c}\text { Trade } \\
\text { Repository }\end{array}$ & $\begin{array}{l}\text { Knowledge } \\
\text { Base }\end{array}$ & $\begin{array}{l}\text { Ontology } \\
\text { Base }\end{array}$ & $\begin{array}{l}\text { Knowledge } \\
\text { Repository }\end{array}$ \\
\hline
\end{tabular}




\subsection{An example of knowledge extraction}

The idea of using ontologies in agent system is to reuse knowledge and direct the agents to share information. Indeed, in a MAS, several agents interact or work together to achieve common goals. [20] Coordination between agents depends on the process and the knowledge they use to achieve their overall goals. The ontology provides a section of the world of knowledge which is essential for the agent to fulfill its tasks.

We present an example of agent knowledge extraction and creation of ontologies.

And here is a Senario:

1) A juridical expert or a wiki administrator builds own ontologies.

2) Then ontologies are Transformed Into queries. These requests are made by the semantic web services. These services can retrieve data from multiple Then databases.

3) The data is Semantically annotated knowledge and Become That Is Stored in year Organizational memory.

4) Users and Learners can access this memory and gain knowledge through research.

\section{S@J-WIKI ARCHITECTURE}

Knowledge agents are some of the cognitive and intelligent agents. They form a coupled network of agents who have worked together to achieve the same goal which is to support the process of knowledge management by providing a comprehensive range of features such as extraction, annotation, storage, update and knowledge sharing[6].
The MAS architecture is a structure of a network of agents with different types of agents and the relationships between them [7].

The architecture of S @ J-Wiki starts from the highest level of abstraction in the description of the companies and agents down to the description of the roles, responsibilities and interactions of agents.

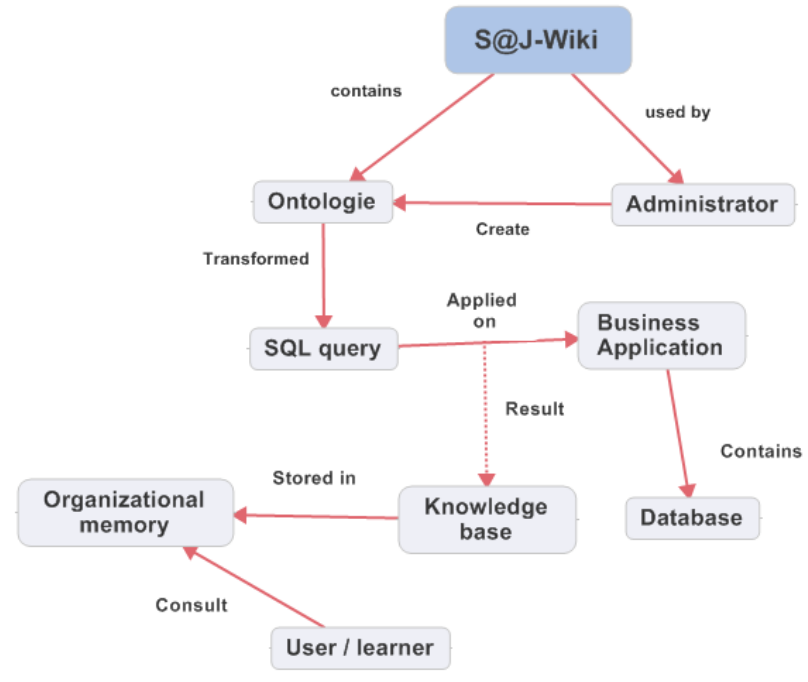

Fig 3: Example of an extraction agent knowledge

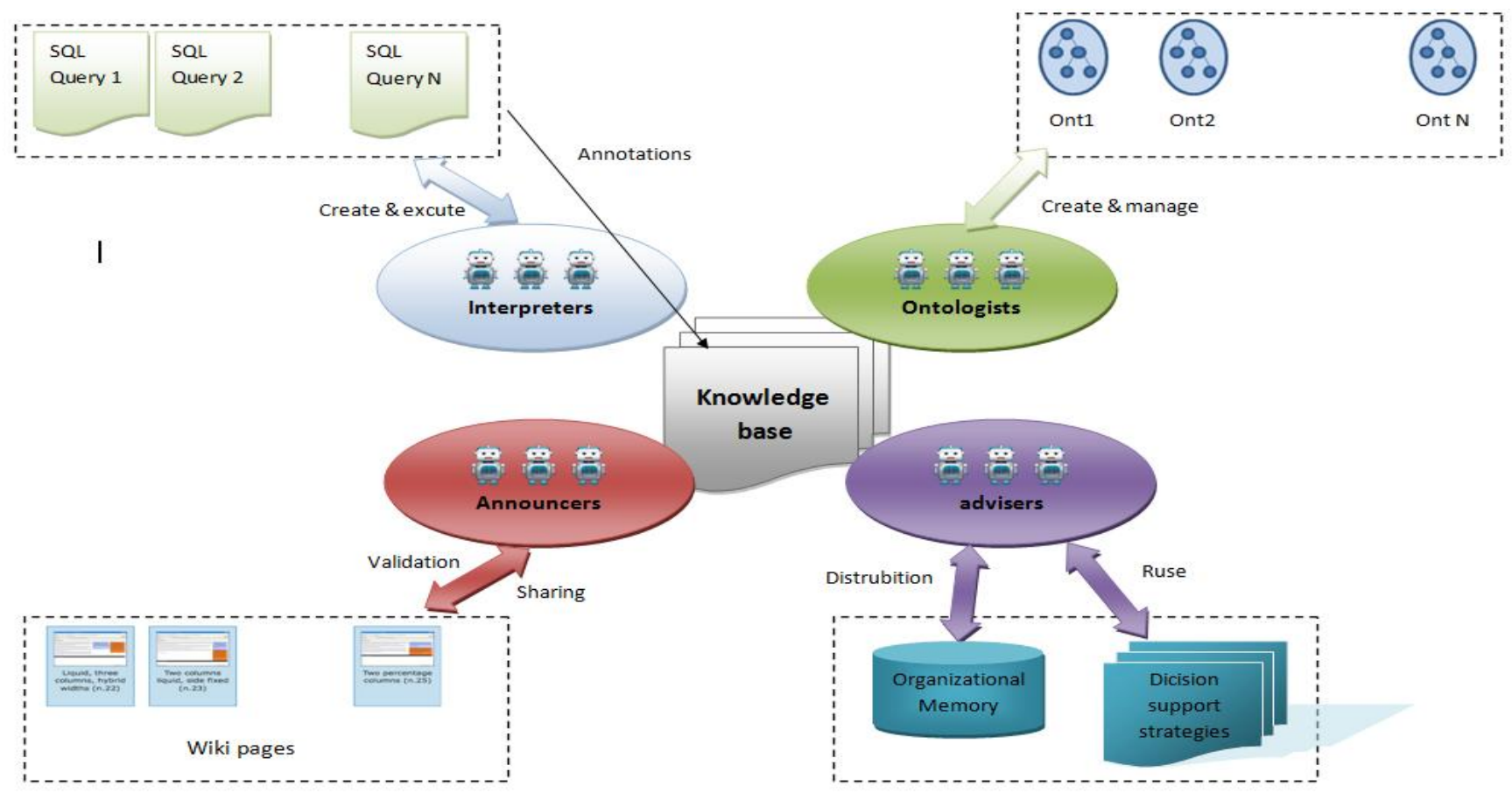

Fig 4 : The S@J-Wiki Architecture 


\subsection{Knowledge definition society "Ontologists"}

The first society of agents, called "ontologists"This society manages different ontologies built by users. The proposed solution can be like Protégé 2000 "ontologists" generate ontologies to OWL-Lite format. It is an ontologist agent for each ontology. The Ontologists also methods manages all ontologies ensuring coherence (ontology alignment process and research of similar concepts) between different ontologies.

\subsection{Knowledge acquisition society "Interpreters"}

The second society of agents is the "interpreters". The role of these agents is to extract knowledge by transforming ontologies models of queries using SQL. Then they consult databases of different sources of information to extract knowledge. Will have one agent for each information source. Interpreters annotate this knowledge by providing an organizational context (creator, the source of information, the domain name, etc). Interpreters are the basis of knowledge with RDF files.

\subsection{Knowledge validation society "Announcers"}

The third society of agents, called "Announcers," the society aims to evaluate, validate and update the knowledge using a semantic wiki. Users can browse knowledge via web wiki pages and can also modify, approve or reject this knowledge. [12]

\subsection{Knowledge reuse society "Advisers"}

The fourth company officers are "advisors". This group of agents are designed to provide decision support for users. He is an advisor for each professional actor. Advisors use a model of organization to monitor the actors' actions. "This model describes the "roles", collaborations and activities during the project users. Thus, asking the knowledge base ie annotations (knowledge and organizational context), advisors can alert and provide users with knowledge that has been stored for a similar activity past, a similar role and a system 'similar information.

\section{IMPLEMENTATION}

In order to provide a platform and evaluation tested for implementation we want develop a distributed multi-agent system for management knowledge. This system is based on the FIPA agent platform JADE [21]. For system configuration there is a domain description formulated in theW3CWeb Ontology Language (OWL). The platform also provides a programming interface to share knowledge among agents based on the domain ontology. Queries are posed in the W3C query language SPARQL.

S @ J-Wiki will be implemented as a run on the Tomcat server using the Jena RDF library for storing metadata Java web application. It uses technology to the date-like AJAX for user interaction.

\section{CONCLUSION AND FUTURE WORK}

This paper presented the architecture of the platform S @ JWiki with the fourths societies agents covering the process of knowledge management. We focused on the extraction of knowledge is performed by interpreters agents. The next step in this research will be to use formal ontologies to extract information and annotate to build a knowledge base in RDF format. In addition we will use an oriented agents agile approach to model our multi-agent system.

\section{REFERENCES}

[1] Jean-Paul BARTHES, Institut International pour 1.Intelligence Artificielle, 1996, UTC, Compiégne

[2] Tim Berners-Lee, James Hendler, Ora Lassila The Semantic Web, Scientific american, May 2001 [http://www.scienfificamerican.com]

[3] Marja-Riitta Koivunen and Eric Miller. W3C Semantic Web Activity. Semantic Web Kick-off Seminar in $\begin{array}{llll}\text { Finland } & \text { Nov } & 2, & 2001\end{array}$ [http://www.w3.org/2001/12/semweb-fin/w3csw]

[4] B. Leuf, W. Cunningham, The Wiki Way: Quick Collaboration on the Web, Addison-Weslay Longmann, 2001.

[5] S. Schaffert, IkeWiki: A Semantic Wiki for Collaborative Knowledge Management, in the 1st International Workshop on Semantic Technologies in Collaborative Applications, STICA, Vol. 6, 2006

[6] R. Lee, T. chen. Revealing research themes and trends in knowledge management: From 1995 to 2010 in Knowledge-Based Systems, December 2011.

[7] F. Gandon, L. Berthelot , Dieng-Kuntz R.,"A MultiAgent Platform for a Corporate Semantic Web",AAMAS 2002, 6th International Conference on Autonomous Agents, 5th International Conference on Multi-Agents Systems, 9th International Workshop on Agent Theories Architectures and Languages Eds Castelfranchi C., Johnson W.L., p. 1025-1032, July 15-19, 2002, Bologna, Italy.

[8] Lahoud, I., Monticolo, D., Gomes, S.: OCEAN: A Semantic Web Service to Extract Knowledge in EGroupwares. In: 6th International Conference on SignalImage Technology.

[9] Abecker A., Bernardi A., and Van Elst L. (2003); Agent technology for distributed organizational memories. In Proceedings of the 5th International Conference On Enterprise Information Systems, Vol. 2, pages 3-10.

[10] Gutknecht O., Ferber J. (2000); Madkit : A generic multiagent platform, AGENTS'00 : 4th International Conference on Autonomous Agents, Barcelona, Spain

[11] Euzenat J., Loup D., Touzani M., Valtchev P., « Ontology Alignement with OLA », Proceedings of the 3rd International Workshop : Semantic Web Conference EON, Hirochima, Japan, p. 341-371, November, 2004 b.

[12] D. Monticolo, S. Gomes "Collaborative Knowledge Evaluation with a Semantic Wiki: WikiDesign", in the International Journal of e-Collaboration, Volume 7, Issue 3. Copyright (C) 2011. 12 pages. 
[13] Corby, O., Dieng-Kuntz, R., Gandon, F., Faron-Zucker, C., 2006. Searching the semantic Web: approximate query processing based on ontologies. IEEE Intelligent Systems 21, 20-27.

[14] Abecker, A., Bernardi, A., Elst, L. van, 2003. Agent Technology For Distributed Organizational Memories: The Frodo Project.

[15] Gandon, F., 2002. Distributed Artificial Intelligence and Knowledge Management: Ontologies and Multi-agent Systems for a Corporate Semantic Web (Thèse).

[16] Bonifacio, M., Bouquet, P., Traverso, P., 2002. Enabling Distributed Knowledge Management: Managerial and Technological Implications.

[17] Jee, K., Yang, J.-J., 2006. Knowledge Description Model for MAS Utilizing Distributed Ontology Repositories, in: Shi, Z.-Z., Sadananda, R. (Eds.), Agent Computing and Multi-Agent Systems, Lecture Notes in Computer Science. Springer Berlin / Heidelberg, pp. 773-780.
[18] Champin, P., Prié, Y., Mille, A., 2003. Musette: Modeling USEs and Tasks for Tracing Experience, in: Workshop 5 "From Structured Cases to Unstructured Problem Solving Episodes For Experience-Based Assistance", ICCBR'03. Trondheim, pp. 279-286.

[19] Monticolo, D., Hilaire, V., Gomes, S., Koukam, A., 2007a. An approach to manage Knowledge based on multi-agents System using a Ontology, in: 19th International Conference on System Research, Informatics \& Cybernetics (InterSymp 2007). Presented at the Symposium on Representation of Context in Software, Baden-Baden, p. 11p.

[20] Rahman, S.A., Yadav, D., Agerwal, P., Bisth, P.S., 2012. Multiagent Knowledge Management Architecture. Journal of Software Engineering and Application 5, 3340

[21] F. Bellifemine, A. Poggi, and G. Rimassa. JADE: A FIPA 2000 compliant agent development environment. In Proceedings of the Fifth International Conference on Autonomous Agents (Agents01). ACM Press, 2001. 